Mathematical Research Letters 1, 465-467 (1994)

\title{
RAMIFIED COVERS OF ABELIAN VARIETIES OVER FUNCTION FIELDS
}

\author{
José Felipe Voloch
}

Lang has conjectured that the set of rational points of a variety of general type defined over a number field is not Zariski dense (see [L]). This has been proved by Faltings $([\mathrm{F} 1,2])$ in the special case that the variety is a subvariety of an abelian variety. The same conjecture can be made for function fields if one excludes isotrivial varieties, i.e. those defined over the constant field. In positive characteristic the conjecture is false since there are non-isotrivial unirational varieties of general type. In characteristic zero, however, the conjecture is plausible. It has also been proved when the variety is a subvariety of an abelian variety $([\mathrm{R}],[\mathrm{B} 1],[\mathrm{H}])$ and when the variety has an ample $\Omega^{1}([\mathrm{~N}],[\mathrm{MD}])$. We add further evidence to the conjecture by proving it for certain ramified covers of abelian varieties over function fields of characteristic zero. Our result is the following:

Theorem. Let $K$ be a function field of characteristic zero and $k$ its constant field. Let $A$ be an abelian variety defined over $K$ with $K / k$-trace zero and $f$ a non-constant rational function on $A$ defined over $K$. Then there exists an integer $n_{0}$ such that, for $n>n_{0}$, the set of $K$-rational points of the cover $X$ of $A$ defined by $z^{n}=f$ is not Zariski dense in $X$.

Proof. [B2], theorem 2, states that there exists $n_{0}$ such that, for any point $P \in A(K)$ and place $v$ of $K / k$, if $f(P)$ is defined and not zero then $\mid v\left(f(P) \mid \leq n_{0}\right.$. To prove the theorem it suffices to show that the rational points of $A$ that lift to rational points of $X$ are not Zariski dense in $A$ and we may consider only those points where $f$ is defined and does not vanish. If $P \in A(K)$ is such a point, lifting to a rational point on $X$, then $f(P)$ is an $n$-th power, so $n \mid v(f(P))$, for any place $v$ of $K / k$ and since $n>n_{0}$, we get, by the above, that $v(f(P))=0$ for all places $v$ of $K / k$, therefore $f(P) \in k$. The theorem now follows from the following proposition, which has independent interest.

Received April 21, 1994.

Research supported by NSF grant DMS-9301157. 
Proposition. Let $K$ be a function field of characteristic zero and $k$ its constant field. Let $A$ be an abelian variety defined over $K$ with $K / k$-trace zero and $f$ a non-constant rational function on $A$ defined over $K$. Then the set $\{P \in A(K) \mid f(P) \in k\}$ is not Zariski dense in $A$.

Proof. Let $G$ be the semi-abelian variety $A \times \mathbf{G}_{m}$ and $Y \subset G$ be the graph of $f$. Let $\Gamma=\left\{\left(P, 2^{n}\right) \in G \mid P \in A(K), n \in \mathbb{Z}\right\}$ and $\Gamma^{*}$ its Kolchin closure. As the Kolchin closure of the group generated by 2 in $\mathbf{G}_{m}$ is $k^{*}$, we get that $\Gamma^{*}$ contains the set $\{(P, x) \in G \mid P \in A(K), x \in k\}$ and to prove the proposition we need to show that $\Gamma^{*} \cap Y$ is not Zariski dense in $Y$. As $\Gamma$ is finitely generated, this follows from the main result of $[\mathrm{H}]$. This completes the proofs of the proposition and the theorem.

Remarks. 1. The proposition is also true in positive characteristic and a proof can be given along the lines of [V], theorem 2, which basically treats the case of elliptic curves.

2. It is not hard to show that covers $X$ of $A$ as in the theorem are of general type for $n$ sufficiently large, provided the support of the divisor of $f$ is ample, which will always be the case if $A$ is simple.

3. I owe the following remark to A. Buium. If $A$ is an abelian variety of dimension at least two and $X$ is a cyclic cover of $A$, branched over a ample divisor with normal crossings, then the irregularity of $A$ and $X$ are the same. This follows from a calculation using the Leray spectral sequence and the Kodaira vanishing theorem. In particular, under these conditions, the Albanese of $X$ is isogenous to $A$ and thus $X$ does not embed in an abelian variety. This applies to most $X$ as in the theorem.

\section{Acknowledgements}

The author would like to thank A. Buium and E. Hrushovski for comments and the NSF for support through the grant DMS-9301157.

\section{References}

[B1] A. Buium, Intersections in jet spaces and a conjecture of S. Lang, Annals of Math. 136 (1992), 557-567.

[B2] A. Buium, The abc conjecture for abelian varieties, Int. Math. Research Notices, to appear.

[F1] G. Faltings, Diophantine approximation on abelian varieties, Ann. Math. 133 (1991), 549-576.

[F2] G. Faltings, The general case of S. Lang's conjecture, Proceedings of the Barsotti conference, to appear.

[H] E. Hrushovski, The Mordell-Lang conjecture for function fields, preprint (1993).

[L] S. Lang, Number theory III: Diophantine Geometry, Encyclopedia of Mathematical Sciences, vol. 60, Springer, Berlin - New York, 1991. 
[MD] M. Martin-Deschamps, Proprietes de descente des varietes a fibre cotangent ample, Ann. Inst. Fourier 34 (1984), 39-64.

[N] J. Noguchi, A higher dimensional analogue of Mordell's conjecture over function fields, Math. Annalen 258 (1981), 207-212.

[R] M. Raynaud, Around the Mordell conjecture for function fields and a conjecture of Serge Lang, Algebraic Geometry, 1-19 (Tokyo / Kyoto 1982); Lecture Notes in Math., vol. 1016, Springer, Berlin - New York, 1983.

[V] J. F. Voloch, A diophantine problem on algebraic curves over function fields of positive characteristic, Bull. Soc. Math. France 119 (1991), 121-126.

Dept. of Mathematics, Univ. of Texas, Austin, Tx 78712 , USA

E-mail address: voloch@math.utexas.edu 